\title{
A combined viscous-vortex, thermal-blob and Lagrangian method for non-isothermal, two-phase flow modelling
}

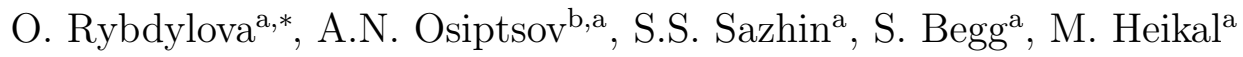 \\ ${ }^{a}$ Sir Harry Ricardo Laboratories, Centre for Automotive Engineering, School of \\ Computing, Engineering and Mathematics, University of Brighton, Brighton BN2 4GJ, \\ $U K$ \\ ${ }^{b}$ Laboratory of Multiphase Flows, Institute of Mechanics, Lomonosov Moscow State \\ University, Moscow 119192, Russia
}

\begin{abstract}
A meshless method for modelling of 2D transient, non-isothermal, gas-droplet flows with phase transitions, based on a combination of the viscous-vortex and thermal-blob methods for the carrier phase with the Lagrangian approach for the dispersed phase, is developed. The one-way coupled, two-fluid approach is used in the analysis. The method makes it possible to avoid the 'remeshing' procedure (recalculation of flow parameters from Eulerian to Lagrangian grids) and reduces the problem to the solution of three systems of ordinary differential equations, describing the motion of viscous-vortex blobs, thermal blobs, and evaporating droplets. The gas velocity field is restored using the Biot-Savart integral. The numerical algorithm is verified against the analytical solution for a non-isothermal Lamb vortex and some asymptotic results known in the literature. The method is applied to modelling of an impulse two-phase cold jet injected into a quiescent hot gas, taking into account droplet evaporation. Various flow patterns are obtained in the calculations, depending on the initial droplet size: (i) low-inertia droplets, evaporating at a higher rate, form ring-like structures and are accumulated only behind the vortex pair; (ii) large droplets move closer to the jet axis, with their sizes remaining almost unchanged; and (iii) intermediate-size droplets are accumulated in a curved band whose ends trail in the periphery behind the head
\end{abstract}

\footnotetext{
*Corresponding author

Email address: 0.Rybdylova@brighton.ac.uk (O. Rybdylova)
} 
of the cloud, with larger droplets being collected at the front of the two-phase region.

Keywords: Droplets, Evaporation, Two-phase flow, Lagrangian approach, Meshless method

PACS: 47.11.-j, 64.70.fm

2000 MSC: $76 \mathrm{~T} 10,80 \mathrm{~A} 20$

\section{Nomenclature}

c $\quad$ specific heat capacity

F mollified kernel

$\mathbf{f}_{d} \quad$ aerodynamic drag force

Fr Froude number

$H \quad$ latent heat of evaporation

$L \quad$ length scale

$l_{\tau} \quad$ droplet velocity relaxation length

$M \quad$ number of thermal blobs

$m_{\text {ref }} \quad$ mass of a droplet of radius $\sigma_{r e f}$

$\dot{m} \quad$ evaporation rate

$N \quad$ number of viscous-vortex blobs

Pr Prandtl number

$q \quad$ heat flux

$\mathbf{r}=(x, y)$ radius vector

Re Reynolds number

$t \quad$ time

T temperature

$U \quad$ velocity scale

$\mathbf{v}=(u, v)$ velocity

\section{Greek symbols}

$\alpha \quad$ thermal expansion coefficient

$\beta \quad$ droplet inertia parameter $\left(\beta=6 \pi \sigma_{\text {ref }} \mu L /\left(m_{\text {ref }} U\right)\right)$

$\Gamma \quad$ velocity circulation, strength of a viscous-vortex blob $\left(\Gamma_{i}=\omega\left(\mathbf{r}_{v i}\right) \Delta_{v i}\right)$

$\gamma \quad$ specific heat ratio $\left(\gamma=c_{p} / c_{v}\right)$

$\Delta_{v i}\left(\Delta_{T i}\right)$ area of a viscous-vortex (thermal) blob

$\delta \quad$ droplet evaporation parameter $\left(\delta=8 \pi \sigma_{\text {ref }} L \lambda\left(T_{\infty}-T_{\text {ref }}\right) /\left(3 m_{\text {ref }} U H\right)\right)$

$\varepsilon \quad$ radius of a blob core 


$\begin{array}{ll}\zeta & \text { cut-off function } \\ \Theta & \text { strength of a thermal blob }\left(\Theta_{i}=T_{0}\left(\mathbf{r}_{T i}\right) \Delta_{T i}\right) \\ \lambda & \text { thermal conductivity } \\ \mu & \text { dynamic viscosity } \\ \rho & \text { density } \\ \sigma & \text { droplet radius } \\ \Psi_{d}, \Psi_{h} & \text { correction functions } \\ \omega & \text { vorticity } \\ & \\ \text { Subscripts } & \\ d & \text { diffusion } \\ i & \text { index } \\ \text { ini } & \text { initial } \\ c & \text { constant pressure } \\ r e f & \text { reference value } \\ s & \text { dispersed phase parameter } \\ T & \text { thermal blob parameter } \\ v & \text { viscous-vortex blob parameter or constant volume } \\ V C & \text { centre of vorticity } \\ 0 & \text { initial value } \\ \infty & \text { value in the far field } \\ \text { Superscripts } & \end{array}$

\section{Introduction}

Meshless methods based on the 'smoothed-particle' approximation proved to be an efficient tool when investigating complex single-phase flows both

5 with primitive and vorticity-velocity variables (Barba et al., 2005; Koumoutsakos, 2005; Andronov et al., 2006; Monaghan, 2012). A combined meshless method for modelling gas-particle flows was considered by Chen and Marshall (1999); Walther and Koumoutsakos (2001). Lebedeva et al. (2013) proposed a method combining the viscous-vortex method for the carrier phase and the Fully La10 grangian approach (Osiptsov, 2000) for particles. This method combines the advantages of both the viscous-vortex and the Fully Lagrangian approaches. It is a fully meshless approach for simulating particle-laden flows, which 
makes it possible to perform accurate calculations of the particle number density fields in transient flows including those with crossing particle trajectories.

The approaches mentioned above were focused on hydrodynamic aspects of particle-laden flows. However, in many engineering applications, including automotive applications (Sazhina et al., 2000; Sazhin et al., 2014), the effects of heat and mass transfer are significant. The objective of the present study is to generalise the approach described in Lebedeva et al. (2013) to take into account some of these effects. The phase transitions on the droplet surface are described using a simple model developed in Osiptsov and Shapiro (1993); Wang and Osiptsov (2002). In this model, the temperature inside the droplets is uniform and its variations with time are negligibly small; the heat flux reaching the droplet is spent only on the evaporation from the droplet surface. This assumption has been widely used for qualitative engineering analysis of droplet evaporation in multiphase flows (e.g. Goldfarb et al. (1999); Sazhin et al. (2010)).

An approach based on the viscous-vortex blob method was chosen in view of its potential application to study the processes in internal combustion engines, accompanying the injection of a jet of fuel. It is recognised that modern vortex methods are well-developed for single-phase flows and can be considered as reasonable alternatives to the Eulerian approach to simulate fluid flows with transient and complex but localised vortex regions (Cottet and Koumoutsakos, 2000). One of the key advantages of these methods is that the calculation of the flow parameters is reduced to solving a system of ordinary differential equations. This implies that the computational time could be optimised if, for example, parallel computing techniques are used (Koumoutsakos, 2005; Sbalzarini et al., 2006). Further advantages of this approach are due to limiting the computational domain only to restricted regions of non-zero vorticity and an absense of an Eulerian grid. Furthermore, the modelling of two-phase flows using the fully meshless (completely Lagrangian) approach avoids the cumbersome 'remeshing' procedure; i.e. the recalculation from the Eulerian to Lagrangian grid. More detailed comparisons between vortex-based and conventional algorithms have been discussed in the literature (Ould-Salihi et al., 2000; Yokota and Obi, 2010).

In the present study, two dimensional, non-isothermal flows are considered and the viscous-vortex blobs are supplemented with 
thermal blobs to take into account the temperature variations in the carrier phase. For $2 \mathrm{D}$ flows, the viscous-vortex blob method has important advantages as the vorticity transport equation may be written in the fully divergence form. This leads to the conservation of the circulation around the boundary of each blob. The same approach is applied to the energy balance equation of the carrier phase. The droplet trajectories and the fields of droplet velocities, temperature, and sizes are calculated using the Lagrangian approach, which makes it possible to calculate correctly regions with intersecting droplet trajectories and to simulate correctly the mixing of droplets in the carrier phase and the spatial variation of droplet sizes due to evaporation. As shown in Lebedeva et al. (2013), the appearance of the regions with crossing trajectories is typical of impulse jet flows, and standard Eulerian approaches fail to describe correctly the distribution of droplet parameters in these regions.

The main aim of the study is to develop a fully meshless approach to simulating two-phase flows with phase transition as an alternative to the conventional Lagrangian-Eulerian approach. The mathematical formulation of the problem is described in Section 2. The details of the numerical method are presented in Section 3. In Section 4, the numerical algorithm is verified based on the comparison of its predictions with the known analytical solution for the Lamb vortex. Section 5 is focused on the numerical simulation of a two-phase jet injection. The main results of the paper are summarised in Section 6 .

We appreciate that the model used in our analysis, particularly the part referring to droplet heating and evaporation, might be too simplistic to allow its direct application to the analysis of realistic engineering processes. However, the approach proposed is expected to lay the basis for further work in this direction, taking into account the realistic polydisperse nature of sprays and using more advanced models for droplet heating and evaporation (Sazhin, 2006, 2014).

\section{Formulation of the problem}

We consider a two-dimensional unsteady flow of a two-phase, gas-droplet mixture with non-uniform temperature distribution taking into account droplet evaporation or condensation. The carrier phase is a viscous heat-conducting gas. The temperature variations in the gas are assumed to be sufficiently small and the flow velocities sufficiently large to neglect the effects of buoy- 
ancy forces and regard the carrier phase as incompressible. The droplets are spheres, treated as a pressureless continuum (Marble, 1970). Due to phase transitions, the radius and mass of the droplets may vary with time. Assuming that the droplet mass loading is small, we neglect the effects of the droplets on the carrier phase. This is a standard assumption of the so-called one-way coupled two-fluid approach, widely used in the literature to study dilute gas-particle mixtures. The range of applicability of such an approach has been widely discussed (e.g. Crowe (2005)). To describe the interphase momentum exchange, the expression for the aerodynamic drag force is used in the form suggested by Carlson and Hoglund (1964). This form takes into account the correction to the Stokes drag for finite inertia effects. Since the ratio of gas to droplet material densities is low $\left(\sim 10^{-2}-10^{-3}\right)$, the contribution of added mass, buoyancy and Basset forces can be neglected. The expression for the heat transfer rate between the gas and the droplets is used in the form suggested by Ranz and Marshall (1952). This expression takes into account the effect of finite values of the Reynolds numbers referring to the flow around a droplet $\left(\operatorname{Re}_{s}\right)$. Thus the force and heat flux on the droplet are presented as follows:

$$
\begin{aligned}
& \mathbf{f}_{d}=6 \pi \sigma^{*} \mu^{*}\left(\mathbf{v}^{*}-\mathbf{v}_{s}^{*}\right) \Psi_{d}, \\
& q_{s}=4 \pi \sigma^{*} \lambda^{*}\left(T^{*}-T_{s}^{*}\right) \Psi_{h},
\end{aligned}
$$

where $\Psi_{d}=1+\operatorname{Re}_{s}^{2 / 3} / 6 ; \Psi_{h}=1+0.3 \operatorname{Pr}^{1 / 3} \operatorname{Re}_{s}^{1 / 2} ; \operatorname{Pr}=c_{p} \mu^{*} / \lambda^{*} ; \operatorname{Re}_{s}=$ $2 \rho^{*} \sigma^{*}\left|\mathbf{v}^{*}-\mathbf{v}_{s}^{*}\right| / \mu^{*}$; the asterisk indicates the dimensional parameters; subscript ' $\mathrm{s}$ ' refers to the dispersed phase parameters; Pr is the Prandtl number; $\rho^{*}, \mu^{*}, \lambda^{*}$, and $c_{p}$ are the gas density, viscosity, thermal conductivity and specific heat at constant pressure, respectively; $\sigma^{*}$ is the droplet radius. More advanced models, including the combined effects of finite $\operatorname{Re}_{s}$ and evaporation on droplet dynamics and heating processes, are discussed in Abramzon and Sazhin (2006); Sazhin (2006, 2014).

The transport and thermodynamic coefficients are assumed to be constant. The phase transition on the droplet surface is assumed to be quasiequilibrium. Accordingly, the droplet surface temperature is related to the vapour pressure by the Clapeyron-Clausius equation. Restricting our analysis to the case of droplets evaporating into their own vapour, there follows that the relative variation of the droplet surface temperature is of the order of the variation in pressure divided by $\rho^{*} H$, where $H$ is the latent heat of evaporation (Wang and Osiptsov, 2002). Since in the flows considered below 
the pressure variations in the flow field are of the order of the pressure head $\rho^{*} U^{2}$, where $U$ is a characteristic flow velocity, the relative variation of the droplet temperature is expected to be of the order of $U^{2} / H$. Considering water droplets or automotive fuel and flow velocities below $100 \mathrm{~m} / \mathrm{s}$, we obtain that $U^{2} / H$ is less than fractions of a percent. Hence, we can expect that the droplet surface temperature is unchanged in the entire region. Note that this temperature can be assumed to be constant in the general case of droplet evaporation into a foreign gas, after the initial stage of the heat-up process has been completed (Sazhin, 2014). We also assume that the temperature inside the droplets is uniform (see Sazhin (2014) for the details).

Under these simplifications, the evaporation rate is controlled by the heat reaching the surface of the droplet:

$$
\dot{m}=\frac{q_{s}}{H} .
$$

The following non-dimensional parameters are introduced:

$$
\begin{aligned}
& \mathbf{r}\left(\mathbf{r}_{s}\right)=\frac{\mathbf{r}^{*}\left(\mathbf{r}_{s}^{*}\right)}{L}, \mathbf{v}\left(\mathbf{v}_{s}\right)=\frac{\mathbf{v}^{*}\left(\mathbf{v}_{s}^{*}\right)}{U}, \\
& T\left(T_{s}\right)=\frac{T_{\infty}-T^{*}\left(T_{s}^{*}\right)}{T_{\infty}-T_{r e f}}, \sigma=\frac{\sigma^{*}}{\sigma_{r e f}}, \\
& \mathbf{r}=(x, y), \mathbf{r}_{s}=\left(x_{s}, y_{s}\right), \mathbf{v}=(u, v), \mathbf{v}_{s}=\left(u_{s}, v_{s}\right),
\end{aligned}
$$

where $L$ and $U$ are the length and velocity scales of the problem; $T_{\text {ref }}$ and $T_{\infty}$ are the gas reference temperature (e.g. jet temperature at the inlet) and the temperature in the far field, respectively, $\sigma_{r e f}$ is the droplet radius scale (e.g. initial droplet radius in the case of initially monodisperse droplets); the so-defined dimensionless temperature takes values between 0 and 1 , with 0 corresponding to the temperature in the far field.

The carrier phase is described by the incompressible Navier-Stokes equations in the vorticity-velocity variables and the transient energy equation in the heat influx form. As mentioned above, the thermal expansion and buoyancy force effects are ignored. We base our analysis on the viscous-vortex and thermal-blob methods (Andronov et al., 2006; Ogami, 1999), in which the dimensionless carrier-phase equations are written in the form:

$$
\begin{aligned}
& \frac{\partial \omega}{\partial t}+\operatorname{div}(\omega \mathbf{v})=\frac{1}{\operatorname{Re} \Delta \omega,} \\
& \frac{\partial T}{\partial t}+\operatorname{div}(T \mathbf{v})=\frac{\gamma}{\operatorname{Re} \operatorname{Pr}} \Delta T,
\end{aligned}
$$


where $\omega=\nabla \times \mathbf{v}$ is the vorticity; $\operatorname{Re}=\rho^{*} L U / \mu^{*}$ and $\gamma=c_{p} / c_{v}$ are the Reynolds number and the specific heat ratio. Equation (3a) is the vorticity transport equation which follows from the Navier-Stokes equations for an incompressible fluid. Equation (3) $)$ is the transient heat conduction equation; under assumption that $U^{2} \ll c_{p}\left(T_{\infty}-T_{r e f}\right)$, the friction dissipation term is ignored.

Introducing the vortex and thermal diffusion velocities, $\mathbf{v}_{d v}$ and $\mathbf{v}_{d T}$, Equations (3) are rewritten in the divergence forms:

$$
\begin{aligned}
& \frac{\partial \omega}{\partial t}+\operatorname{div}\left(\omega\left(\mathbf{v}+\mathbf{v}_{d v}\right)\right)=0 \\
& \frac{\partial T}{\partial t}+\operatorname{div}\left(T\left(\mathbf{v}+\mathbf{v}_{d T}\right)\right)=0
\end{aligned}
$$

where

$$
\mathbf{v}_{d v}=-\frac{1}{\operatorname{Re}} \frac{\nabla \omega}{\omega}, \mathbf{v}_{d T}=-\frac{\gamma}{\operatorname{Re} \operatorname{Pr}} \frac{\nabla T}{T} .
$$

System (4) describes the evolution of the vorticity and temperature fields.

Assuming that the flow domain is unbounded and the finite-vorticity and nonuniform temperature regions are bounded in space, the initial and boundary conditions for System (4) can be formulated as:

$$
\begin{aligned}
& \omega\left(\mathbf{r}, t_{0}\right)=\omega_{0}(\mathbf{r}), \\
& T\left(\mathbf{r}, t_{0}\right)=T_{0}(\mathbf{r}), \\
& \mathbf{r} \rightarrow \infty: \omega \rightarrow 0, T \rightarrow 0 .
\end{aligned}
$$

Once the vorticity field is calculated, then the velocity field can be restored using the Biot-Savart integral.

The dispersed-phase dimensionless kinematic, momentum balance and evaporation (2) equations can be written in the Lagrangian form:

$$
\begin{aligned}
& \frac{d \mathbf{r}_{s}}{d t}=\mathbf{v}_{s}, \\
& \frac{d \mathbf{v}_{s}}{d t}=\frac{\beta}{\sigma^{2}}\left(\mathbf{v}-\mathbf{v}_{s}\right) \Psi_{d}, \\
& \frac{d \sigma^{2}}{d t}=\delta\left(T-T_{s}\right) \Psi_{h},
\end{aligned}
$$


where

$$
\begin{aligned}
& \beta=\frac{6 \pi \sigma_{r e f} \mu^{*} L}{m_{r e f} U}, \delta=\frac{8 \pi}{3} \frac{\sigma_{r e f} L \lambda^{*}\left(T_{\infty}-T_{r e f}\right)}{m_{r e f} U H}, \\
& \operatorname{Re}_{s}=\operatorname{Re}_{s 0} \sigma\left|\mathbf{v}-\mathbf{v}_{s}\right|, \operatorname{Re}_{s 0}=\frac{2 \rho^{*} \sigma_{r e f} U}{\mu^{*}}
\end{aligned}
$$

Here, $\beta, \delta, \operatorname{Re}_{s 0}$ are the droplet inertia parameter (inverse to the Stokes number (Marble, 1970)), evaporation parameter and the Reynolds number of the flow around a droplet; $m_{r e f}$ is the mass of a droplet of radius $\sigma_{r e f}$.

The initial conditions for System (5) are as follows:

$$
\begin{aligned}
& t=t_{0}: \mathbf{r}_{s}=\mathbf{r}_{0}, \mathbf{v}_{s}=\mathbf{v}_{s 0} \\
& T_{s}=T_{s 0}(\mathbf{r}), \sigma=\sigma_{0}(\mathbf{r})
\end{aligned}
$$

The flow of the 'gas-evaporating droplet' system is controlled by six similarity parameters: the flow Reynolds number Re, the Prandtl number Pr, the specific heat ratio $\gamma$, the droplet inertia and evaporation parameters ( $\beta$ and $\delta)$, and the characteristic droplet Reynolds number $\mathrm{Re}_{s}$.

\section{Numerical algorithm}

The carrier phase parameters are calculated using the meshless vortex and thermal blob methods, while the Lagrangian approach is used for calculating the dispersed phase parameters. We focus here only on the basics of the numerical algorithm used in the vortex and thermal blob methods; see (Ogami, 1999; Cottet and Koumoutsakos, 2000; Andronov et al., 2006) for further details.

Consider a bounded domain with a non-zero vorticity. This domain can be discretised into $N$ elements, with the area of the $i$-th element equal to $\Delta_{v i}$. Thus:

$$
\begin{aligned}
& \omega(\mathbf{r}, t) \approx \sum_{i=1}^{N} \Gamma_{i} \zeta_{\varepsilon_{i}}\left(\mathbf{r}-\mathbf{r}_{v i}(t)\right), \\
& \Gamma_{i} \approx \omega_{0}\left(\mathbf{r}_{v i}\left(t_{0}\right)\right) \Delta_{v i}=\mathrm{const},
\end{aligned}
$$

where $\zeta_{\varepsilon_{i}}(\mathbf{r})$ are the so-called cut-off functions. The elements of discretisation are called blobs. As follows from System (41), the blobs' strengths $\Gamma_{i}$ (equal 
to the initial velocity circulations along the blob contours as described by the Stokes' theorem) are conserved along the trajectory described by the velocity $\mathbf{v}+\mathbf{v}_{d v}$. The specific form of the cut-off functions $\zeta_{\varepsilon_{i}}$, describing the interpolation between blobs, is discussed later in this section. The current viscous-vortex blob positions $\mathbf{r}_{v i}$ are calculated from the system of ordinary differential equations:

$$
\frac{d \mathbf{r}_{v i}}{d t}=\mathbf{v}\left(\mathbf{r}_{v i}, t\right)+\mathbf{v}_{d v}\left(\mathbf{r}_{v i}, t\right)
$$

The equations, similar to (66) and (77) but for $M$ thermal blobs, take the form:

$$
\begin{aligned}
& T(\mathbf{r}, t) \approx \sum_{i=1}^{M} \Theta_{i} \zeta_{\varepsilon_{i}}\left(\mathbf{r}-\mathbf{r}_{T i}(t)\right), \\
& \Theta_{i}=T_{0}\left(\mathbf{r}_{T i}\left(t_{0}\right)\right) \Delta_{T i}=\mathrm{const}, \\
& \frac{d \mathbf{r}_{T i}}{d t}=\mathbf{v}\left(\mathbf{r}_{T i}, t\right)+\mathbf{v}_{d T}\left(\mathbf{r}_{T i}, t\right),
\end{aligned}
$$

where $\Theta_{i}$ and $\mathbf{r}_{T i}$ are the strength and position of the $i$-th thermal blob.

The accuracy of calculations depends on the choice of the form of the cut-off function $\zeta_{\varepsilon_{i}}(\mathbf{r})$. In contrast to Lebedeva et al. (2013), where a very simple cut-off function was used, we used the 4th order cut-off function, recommended in Cottet and Koumoutsakos (2000):

$$
\zeta_{\varepsilon_{i}}(\mathbf{r})=\frac{1}{3 \pi \varepsilon_{i}^{2}}\left(4-\frac{\mathbf{r}^{2}}{\varepsilon_{i}^{2}}\right) \exp \left(-\frac{\mathbf{r}^{2}}{\varepsilon_{i}^{2}}\right)
$$

Here, $\varepsilon_{i}$ is the core radius of the $i$-th blob (parameter associated with the cut-off function $\left.\zeta_{\varepsilon_{i}}(\mathbf{r})\right)$. Note that the choice of the $\varepsilon_{i}$ affects the accuracy of the results. The values of $\varepsilon_{i}$ are discussed in the following sections. In a general case, the spatial discretisation of the initial domain into the viscous blobs differs from that for the thermal blobs.

As follows from (6) and (8), the diffusion velocities are calculated using 
the following formulae (Andronov et al., 2006; Ogami, 1999)

$$
\begin{gathered}
\mathbf{v}_{d v}=-\frac{1}{\operatorname{Re}} \frac{\sum_{i=1}^{N} \Gamma_{i} \cdot \nabla \zeta_{\varepsilon_{i}}\left(\mathbf{r}-\mathbf{r}_{v i}(t)\right)}{\sum_{i=1}^{N} \Gamma_{i} \zeta_{\varepsilon_{i}}\left(\mathbf{r}-\mathbf{r}_{v i}(t)\right)}, \\
\mathbf{v}_{d T}=-\frac{\gamma}{\operatorname{Re} \operatorname{Pr}} \frac{\sum_{i=1}^{M} \Theta_{i} \cdot \nabla \zeta_{\varepsilon_{i}}\left(\mathbf{r}-\mathbf{r}_{T i}(t)\right)}{\sum_{i=1}^{M} \Theta_{i} \zeta_{\varepsilon_{i}}\left(\mathbf{r}-\mathbf{r}_{T i}(t)\right)} .
\end{gathered}
$$

The velocity field is calculated using the Biot-Savart integral (Andronov et al., 2006) and the mollified kernel of the 4-th order (Cottet and Koumoutsakos, 2000):

$$
\begin{aligned}
\mathbf{v}(\mathbf{r}) & =\frac{1}{2 \pi} \sum_{i=1}^{N} \Gamma_{i} \mathbf{e}_{z} \times \frac{\mathbf{r}-\mathbf{r}_{v i}}{\left|\mathbf{r}-\mathbf{r}_{v i}\right|^{2}} \approx \\
& \approx \frac{1}{2 \pi} \sum_{i=1}^{N} \Gamma_{i} \mathbf{e}_{z} \times \mathbf{F}_{\varepsilon_{i}}\left(\mathbf{r}-\mathbf{r}_{v i}\right), \\
\mathbf{F}_{\varepsilon_{i}}(\mathbf{r}) & =\frac{\mathbf{r}}{2 \pi \mathbf{r}^{2}}\left[1+\left(\frac{\mathbf{r}^{2}}{\varepsilon_{i}^{2}}-1\right) \exp \left(-\frac{\mathbf{r}^{2}}{\varepsilon_{i}^{2}}\right)\right] .
\end{aligned}
$$

At each time step, Equations (77) for all viscous-vortex blobs (indicated by subscript ' $i$ ') are solved. Then for each thermal blob, Equations (9) are solved using Expressions (10) to compute the fluid velocity at each point $\mathbf{r}_{T i}(t)$. Finally, the droplet parameters are calculated from (5) with the values of the carrier phase velocity and temperature at any point $\mathbf{r}_{s}(t)$ obtained using (10) and (8). The systems of ordinary differential equations (7), (9), and (15) are solved using the 4 -th order Runge-Kutta method (Boost C++ Libraries v. 1.53, 2014).

To summarise, the numerical algorithm under consideration comprises three components: viscous-vortex, thermal-blob and droplet dynamics and evaporation. The first and the second components will be verified against analytical solutions. The values of the numbers of thermal blobs, $M$ and viscous-vortex blobs, $N$ vary for different problems in the general case and an investigation of the 
accuracy of the calculations of the carrier phase parameters should be carried out for each specific problem. In our study, the starting values of the numbers of thermal blobs and viscous-vortex blobs are chosen such that the results are found to be independent of both $M$ and $N$.

For the low Mach number flows considered, the effects of compressibility are ignored as the temperature variation is relatively small and the Froud number is relatively high $\left(\alpha \Delta T^{*} / \operatorname{Fr}^{2} \sim 10^{-2}\right.$, where $\alpha$ is the thermal expansion coefficient, $\Delta T^{*}$ is the temperature difference, $\mathrm{Fr}$ is the Froude number). In this case the buoyancy force in the Boussinesq approximation can be neglected.

The method developed is based on a simple evaporation model, which does not take into account the contribution of the heatingup period and the vapour concentration distribution. In order to implement more advanced models, the equation for the vapour concentration diffusion must be included as well. This equation can be rewritten using 'diffusion diffusion' velocity as in (44) and solved after introducing 'diffusion blobs'. However, this analysis is beyond the scope of the paper.

\section{Verification of the numerical method: Lamb vortex}

The accuracy of calculations depends on a number of parameters entered into the discretisation formulas (the numbers of viscous-vortex and thermal blobs ( $\mathrm{N}$ and $\mathrm{M}$ ), the initial geometry of the blobs, the time step used in calculating the systems of ordinary differential equations, and the blob overlapping radii $\varepsilon_{i}$.)

To verify our numerical algorithm and to choose the appropriate values of the above-listed parameters, we used a standard benchmark for testing numerical codes based on the vortex methods, namely, the Lamb vortex flow described by an exact analytical solution to the transient Navier-Stokes equations.

Let us introduce a Cartesian coordinate system with the origin at the vortex centre. Then the vorticity and velocity fields for this vortex are given 
by the following formulae (Batchelor, 1967):

$$
\left\{\begin{array}{l}
\omega=\frac{\operatorname{Re}}{4 \pi t} \exp \left(-\operatorname{Re} \frac{\mathbf{r}^{2}}{4 t}\right), \\
\mathbf{v}=(u, v)=\frac{(-y, x)}{2 \pi \mathbf{r}^{2}}\left[1-\exp \left(-\operatorname{Re} \frac{\mathbf{r}^{2}}{4 t}\right)\right],
\end{array}\right.
$$

where the Reynolds number is based on the initial velocity circulation $\Gamma_{0}$ : $\operatorname{Re}=\rho \Gamma_{0} / \mu$. In Expressions (111) the length scale is taken equal to the droplet velocity relaxation length $l_{\tau}=\sqrt{m_{r e f} \Gamma_{0} /\left(6 \pi \sigma_{r e f} \mu\right)}$, and the velocity scale $U=\Gamma_{0} / l_{\tau}$. This choice of the length and velocity scales corresponds to $\beta=1$.

The initialisation of the viscous-vortex blobs was the same as in Lebedeva et al. (2013), except that the blobs were placed on concentric circumferences, with the radii increasing with a fixed step $\Delta r=0.025$ (rather than the step decreasing towards the vortex centre). This leads to a series of rings, with the blobs distributed uniformly in each ring. The strength of the blobs was calculated as the difference in the circulations along the outer and inner radii at $t=t_{0}$ divided by the number of blobs in the ring. Since Solution (11) has a singularity at $t=0$, the initial time instant was taken as $t_{0}=1$. Increasing the number of blobs in the calculations, we found that a satisfactory accuracy could be achieved with 863 viscous-vortex blobs, and a core size $\varepsilon=c_{\varepsilon} \sqrt{h}$, where $h$ is the width of the corresponding ring, $c_{\varepsilon}=0.7$ (Barba et al., 2005). For this number of blobs our algorithm gives satisfactory results not only for the velocity field but also for the derivatives of the velocity. Fig. 1a shows a comparison of the predictions of the exact analytical and numerical solutions at various time instants for $\mathrm{d}^{2} u / \mathrm{d} t \mathrm{~d} y$ at the $y=0$ cross-section, $\operatorname{Re}=100$. The closeness of the plots shows that our numerical code is expected to be reliable and that it can be used for the analysis of other flows.

In the next step, we verified the thermal part of our algorithm. Equation (3b) is equivalent to Equation (3a), if $\gamma=\operatorname{Pr}$. Hence, in this case, an exact solution for the temperature field evolution, corresponding to the diffusion of the localised temperature non-uniformity in the Lamb vortex field, can be described as:

$$
T=\frac{\operatorname{Re}}{4 \pi t} \exp \left(-\operatorname{Re} \frac{\mathbf{r}^{2}}{4 t}\right)
$$

Expression (12) was used to verify the calculations of the temperature field distribution. The initial location of thermal blobs was the same as that of 
the viscous-vortex blobs. For $\mathrm{Re}=100$, the initial temperature distribution was set as $T=25 / \pi \exp \left(-25 r^{2}\right), t_{0}=1$. The strength of each thermal blob is the temperature at the blob centre, multiplied by the blob area (the area of a ring divided by the number of blobs in the ring). The temperature profiles at the $y=0$ cross-section at various time instants against the predictions of the analytical solution are presented in Fig. 1 b. As in the case of the vorticity field, the results were shown not to depend on the number of thermal blobs when this number exceeded 863. The latter number of thermal blobs has been used in our analysis.

Then the droplet parameters were calculated from the solution to System (5). The droplet evaporation was calculated for $\operatorname{Re}=100, \operatorname{Pr}=0.8$, $\gamma=1.33, \operatorname{Re}_{s 0}=0, \delta=-0.1$. It was assumed that the heat flux is directed towards the droplets. Hence, only evaporation takes place. The initial temperature distribution was set as $T=\exp \left(-r^{2} / 0.04\right)$, which implies that $0<T \leq 1$. Since the problem under study is axially symmetric, the fields of the droplet parameters are presented as functions of the distance to the axis of symmetry. The initial conditions for the dispersed phase are the following:

$$
\begin{aligned}
& t=t_{0}: \mathbf{r}_{s}=\mathbf{r}_{0}, \mathbf{v}_{s}\left(\mathbf{r}_{s}\right)=\mathbf{v}\left(\mathbf{r}_{s}\right), \\
& T_{s}(x, y)=0, \sigma=1 .
\end{aligned}
$$

Inertial droplets tend to move away from the centre of the vortex. The droplets initially positioned closer to the vortex centre have higher initial velocity and they overtake the droplets initially located at the periphery. The trajectory intersections result in the formation of a multivalued field of droplet parameters (see Figs. 3 and 4 at $t=3, t=4, t=5$ ). In Fig 3 , the time and space evolution of droplet radii in the Lamb vortex are shown in the range from $t=1$ to $t=5$. The central region of the flow corresponds to the highest difference in the temperatures between the carrier and the dispersed phases, which leads to the highest evaporation rates. As mentioned earlier, the direct effect of the evaporation process on the drag force coefficient is not taken into account (see (Abramzon and Sazhin, 2006) for the details). However since the droplets evaporate, the drag force exerted on them reduces, as their radii decrease. This effect is shown in Fig. 4, where the temporal evolutions of $\left|\mathbf{v}_{s}\right|$ and $\mathbf{r}_{s}$ of a line of droplets, placed in the Lamb vortex, are compared for the cases of evaporating and non-evaporating droplets for $t$ in the range $t=1$ to $t=5$. The dotted curve in Fig. 4 corresponds to the analytical solution (11). As droplets evaporate, their inertia decreases; 


\begin{tabular}{ccc}
\hline$\sigma^{*}, \mu \mathrm{m}$ & $\beta$ & $\delta$ \\
\hline 100 & 0.05 & 0.0014 \\
50 & 0.21 & 0.006 \\
10 & 5.5 & 0.14 \\
\hline
\end{tabular}

Table 1: Dimensionless values of the droplet parameters

the droplet velocities are closer to those of the carrier phase in the case of evaporating droplets.

\section{Impulse two-phase jet flow}

In this section, the approach described above is applied to the simulation of the injection of a cold, two-phase jet into a hot, quiescent gas. In the case of an impulse jet with a step-like velocity distribution, a vortex ring (or vortex pair) is usually formed after the jet injection (Gharib et al., 1998). The study presented in this section is focused on the formation and dynamics of a two-phase vortex pair.

We consider the injection of a water spray with $L=0.01 \mathrm{~m}$ (nozzle width), $U=1.5 \mathrm{~m} / \mathrm{s}, T_{\infty}-T_{\text {ref }}=50 \mathrm{~K}$ into water vapour. The dimensionless parameters in this case are summarised in Table 1. In the two-phase jet flow, small droplets have low inertia, and are easily entrained in the vortex flow. Droplets with larger inertia form a different flow pattern, since they travel with a noticeable velocity slip relative to the carrier phase. Three cases corresponding to droplets with different inertia were considered in order to illustrate different flow regimes. The Cartesian coordinate system is introduced: $\mathrm{x}$-axis is directed along the jet axis of symmetry, the origin is at the inlet of the jet. The calculation results are presented in Figs. 5-10 for $\operatorname{Re}=1000, \operatorname{Pr}=0.8, \gamma=1.33, \operatorname{Re}_{s 0}=0$, (i) $\beta=0.05, \delta=0.0014$, (ii) $\beta=0.21, \delta=0.006$, (iii) $\beta=5.5, \delta=0.14$.

In the calculations, the initial dimensionless vorticity distribution corresponded to a smooth step-like velocity profile taken in the form:

$$
\left\{\begin{array}{l}
u_{0}=\frac{1}{1+\exp (-100(y+0.4))}+\frac{1}{1+\exp (100(y-0.4))}-1, \\
v_{0}=0
\end{array}\right.
$$

The initial dimensionless temperature distribution was taken as $T_{0}(x, y)=$ $u_{0}(x, y)$. The initial conditions for the dispersed phase were assumed to be 
the following:

$$
\begin{aligned}
& t=t_{0}: \mathbf{r}_{s}=\mathbf{r}_{0}, \mathbf{v}_{s}=\left(u_{s 0}, v_{s 0}\right)=(0.8,0) \\
& T_{s}(x, y)=1, \sigma=1
\end{aligned}
$$

The vorticity inferred from the velocity distribution described by System (13) is zero in the most part of the domain, including the axis $y=0$, except for the two small regions at negative and positive $y$. This allows us to initially position the viscous blobs in two rectangles $[-4 ; 0] \times[0.24 ; 0.56]$ and $[-4 ; 0] \times[-0.56 ;-0.24]$. The thermal blobs are located initially in the rectangle $[-4 ; 0] \times[-0.5 ; 0.5]$. The domain geometry $(D / L=4)$ corresponds to the vortex pair 'formation number' (Gharib et al., 1998). The droplets occupy the central part of the domain, the rectangle $[-4 ; 0] \times[-0.4 ; 0.4]$. At the initial time instant, the regions on both sides of the injected liquid column are free of droplets, and the droplet velocities are $20 \%$ less than that of the carrier phase. At the initial instant of time, all blobs and droplets were distributed uniformly. The strength of a blob was calculated as the product of the corresponding value of the parameter (vorticity or temperature) and the blob area. The variable core size for each blob was calculated using the formulae recommended in Barba et al. (2005):

$$
\varepsilon_{i}=c_{\varepsilon} \min _{j=\overline{1, N}}\left(\sqrt{\left|\mathbf{r}_{v j}-\mathbf{r}_{v i}\right|}\right)
$$

for viscous-vortex blobs, and

$$
\varepsilon_{i}=c_{\varepsilon} \min _{j=\overline{1, M}}\left(\sqrt{\left|\mathbf{r}_{T j}-\mathbf{r}_{T i}\right|}\right),
$$

for thermal blobs, where $c_{\varepsilon}=1.01$.

After the injection, the viscous-vortex blobs concentrated in two spirals, being swirled in the opposite directions and propagating into the quiescent gas. The initial locations and velocities of thermal blobs differ from those of viscous-vortex blobs. Hence, they fill a region different from the one occupied by the viscous-vortex blobs. The instant locations of all the blobs at $t=5$ and $t=14$ (10000 viscous vortex and 10000 thermal blobs) are presented in Fig. 5. Large grey circles correspond to the vortex blobs; different levels of shading indicate different values of blob strength; black dots refer to the thermal blobs. 
The numerical results for the carrier phase velocity fields were compared with the results predicted by the asymptotic analytical solution to the problem of the diffusion of a vortex pair (Gaifullin and Zubtsov, 2004), according to which the 'centres' of the vorticity regions travel with the velocity:

$$
V_{V C}\left(\frac{t^{*} \Gamma_{i n i}}{l_{0}^{2}}\right)=\frac{\Gamma_{i n i}}{4 \pi l_{0}}\left[1-8.736\left(\frac{t^{*} \nu}{l_{0}^{2}}\right)^{2}+1.845\left(\frac{t^{*} \nu}{l_{0}^{2}}\right)^{3}\right] .
$$

The asymptotic solution (14) corresponds to the limit of high Reynolds numbers $\operatorname{Re}_{a}=\Gamma_{i n i} / \nu>>1, \Gamma_{i n i}$ is the intensity of each of the vortices at the initial time instant, and $\pm l_{0}$ are the initial ordinates of the vortices. Since the vortex pair is symmetric, only the top vortex position was calculated using the formula:

$$
\begin{aligned}
& \Gamma_{\text {top }}=\sum_{i: y_{v i}>0} \Gamma_{i} \\
& \mathbf{r}_{V C}=\frac{1}{\Gamma_{\text {top }}} \sum_{i: y_{v i}>0} \mathbf{r}_{v i} \Gamma_{i}
\end{aligned}
$$

The reference values for the vortex center position and velocity were taken at $t=4$; for the analytical solution (14) the initial time instant was taken equal to $t_{0}=2.6$. In Fig. [6 a half-distance between the vortices, calculated using 10000, 6400, and 3600 viscous-vortex blobs, is compared with the asymptotic solution. Satisfactory accuracy was obtained when 10000 viscous-vortex blobs were used for the calculations. The results presented in Figures 6-10 were obtained for 10000 viscous-vortex blobs, 10000 thermal blobs, and 1681 droplets.

The 'thermal centres' were calculated using the formula (cf. (15)):

$$
\begin{aligned}
& \Theta_{\text {top }}=\sum_{i: y_{v i}>0} \Theta_{i} \\
& \mathbf{r}_{T C}=\frac{1}{\Theta_{\text {top }}} \sum_{i: y_{v i}>0} \mathbf{r}_{T i} \Theta_{i}
\end{aligned}
$$

The positions of the vortex and thermal centres, calculated numerically, are compared with the predictions of the asymptotic solution 14 (for vortex centre) in Fig. 6b. As follows from this figure, the 'thermal centre' is located closer to the jet axis. This is due to the fact, that the viscous-vortex blobs 
are all involved in the spiral motion; whereas thermal blobs form spirals and characteristic tail features (see Fig. 5, $t=14$ ).

Vortex centre dynamics predicted by the numerical simulation were compared with the prediction of the asymptotic solution (14) for the cases of $\operatorname{Re}=100$ and $\operatorname{Re}=1000$. The positions of the vortex pair centres are presented in Fig. 7. As follows from this figure, the asymptotic solution $(\mathrm{Re} \rightarrow \infty)$ predicts slightly higher values than the numerical simulations; there is a better agreement between the results for the case of $\operatorname{Re}=1000$. In both cases the numerical results agree satisfactorily with the predictions of the analytical solution at the initial times $(0<t<10)$; at later times the discrepancy increases. This discrepancy becomes particularly noticeable in the case of $R e=100$ as the analytical solution was derived in the limit of infinitely high Reynolds numbers of the flow.

The cases of droplets with $\beta=5.5, \beta=0.21$, and $\beta=0.05$ were considered (see Table 1). In Fig. 8-10, clouds of droplets at various time instants are presented. Due to their symmetry, only one half of the droplet flow pattern is shown. In the top parts of the figures, the highest level of shading corresponds to the largest droplets, and the lowest level to the smallest droplets. In the bottom parts of the figures, the highest level of shading corresponds to the largest phase velocity slips, and the lowest level to the smallest velocity difference. As one can see in these figures, after the injection, a vortex pair forms in the carrier phase. The velocities of low-inertia particles $(\beta=5.5$, see Fig. 8) relax faster than the carrier phase velocities. Thus, the two-phase flow is characterised by better mixing; the droplets form ring-like structures and evaporate faster compared with the cases of larger droplets. At $t=8$, smaller droplets are located closer to the jet axis, while larger droplets can be seen at the periphery. This is attributed to the fact that, as droplets enter the hot environment, they evaporate faster and are entrained in the vortex earlier than the droplets which enter the flow at later times. At $t=12$, droplets remain behind the vortex, as large droplets move backwards and small droplets evaporate completely. The case $\beta=0.21$ (see Fig. 9) corresponds to the widest two-phase jet among the cases considered. The velocities of the droplets, that were initially close to the inlet, decrease as they enter the quiescent gas; the droplets that enter the flow later can overtake the leading edge. The initially rectangular cloud of droplets is turned inside out after the injection, then it is deformed into a narrow band (see $t=8$, $t=12$ ). The droplets at the sides move slower and evaporate more rapidly 
than those at the centre. As the droplets at the front of the two-phase region evaporate and slow down, larger droplets move to the front edge $(t=12)$. At later times the cloud of droplets is divided into two groups, positioned along the jet axis. The droplet sizes vary within a $7 \%$ range. In the case of droplets with greater inertia ( $\beta=0.05$, see Fig. 10), their sizes remain almost unchanged. The cloud of droplets deforms into a narrow band. The droplets evaporate at a greater rate at the front of the two-phase region. Hence, larger droplets remain at the rear part of the droplet cloud. The two-phase region remains closer to the jet axis.

These results are in good qualitative agreement with droplet behaviour observed in experiments. Begg et al. (2009) investigated vortex ring-like structures visualised by fuel droplets in a combustion chamber. It was shown that, in this flow, the largest droplets move faster along the axis of the vortex ring, and the smallest droplets are entrained in vortex rotation. It was also shown that the axial velocity of droplets on the axis is higher than that off the axis.

\section{Conclusion}

A meshless method based on a combination of the viscous-vortex and thermal-blob methods for the carrier phase and the Lagrangian approach for the dispersed phase is developed for the case of non-isothermal flow and evaporating droplets. The effect of the droplets on the carrier phase was not considered. Some numerical results referring to transient $2 \mathrm{D}$ gas-droplet flows are presented. The numerical code was verified for the non-isothermal Lamb vortex flow. The predicted distributions of the carrier-phase parameters were shown to be in good agreement with the analytical solution for the Lamb vortex. The method was applied to simulate a plane impulse two-phase jet flow. The injection of a cold 'vapour-droplet' jet into a hot quiescent gas was studied. The calculated parameters of the vortex pair formed after the injection are shown to be in good agreement with the known asymptotic solution for the diffusion of two vortices. Three typical cases corresponding to different initial droplet diameters were considered. The flow with the finest droplets shows better mixing among the three cases considered: low-inertia droplets form ring-like structures. The droplets of medium size collect into narrow bands, the droplets at the band ends slow down and evaporate more rapidly than at the centre. The cloud of droplets with the largest inertia 
remain close to the jet axis. This result was shown to be supported by experimental observations. In the three cases considered, the evaporation process leads to different droplet size distributions in the flow field: (i) in the case of small droplets, larger droplets collect behind the vortex pair; (ii) in the case of medium size droplets, larger droplets accumulate at the front of the two-phase jet; (iii) in the case of the largest droplets, larger droplets accumulate at the trailing part of the two-phase region.

\section{Acknowledgements}

The authors are grateful to the EPSRC (grants EP/K005758/1 and EP/M002608/1) (UK) and the Russian Foundation for Basic Research (RFBR) (No. 14-0100147) for financial support and to Dr. N.A. Lebedeva for helpful discussions.

\section{References}

Abramzon, B., Sazhin, S. S., 2006. Convective vaporization of a fuel droplet with thermal radiation absorption. Fuel 85 (1), $32-46$.

Andronov, P. R., Guverniuk, S. V., Dynnikova, G. Y., 2006. Vortex Methods for Calculation of Transient Hydrodynamic Loads. Moscow State University, Ch. 1, [in Russian].

Barba, L. A., Leonard, A., Allen, C. B., 2005. Advances in viscous vortex methods-meshless spatial adaption based on radial basis function interpolation. International Journal for Numerical Methods in Fluids 47 (5), $387-421$

Batchelor, G., 1967. An Introduction to Fluid Mechanics. Cambridge University Press.

Begg, S., Kaplanski, F., Sazhin, S. S., Hindle, M., Heikal, M., 2009. Vortex ring-like structures in gasoline fuel sprays under cold-start conditions. International Journal of Engine Research 10 (4), 195-214.

Boost C++ Libraries v. 1.53, 2014.http://www.boost.org/users/history/version_1_53_0. accessed: 26.10 .2014 . 
Carlson, D. J., Hoglund, R. F., 1964. Particle drag and heat transfer in rocket nozzles. AIAA Journal 2 (11), 1980-1984.

Chen, H., Marshall, J., 1999. A Lagrangian vorticity method for two-phase particulate flows with two-way phase coupling. Journal of Computational Physics 148 (1), 169 - 198.

Cottet, G.-H., Koumoutsakos, P., Jun. 2000. Vortex methods: Theory and practice. Cambridge University Press, Ch. 2.

Crowe, C. T., 2005. Multiphase Flow Handbook. CRC Press, Ch. 1.

Gaifullin, A., Zubtsov, A., 2004. Diffusion of two vortices. Fluid Dynamics 39 (1), 112-127.

Gharib, M., Rambod, E., Shariff, K., 4 1998. A universal time scale for vortex ring formation. Journal of Fluid Mechanics 360, 121-140.

Goldfarb, I., Gol'dshtein, V., Kuzmenko, G., Sazhin, S. S., 1999. Thermal radiation effect on thermal explosion in gas containing fuel droplets. Combustion Theory and Modelling 3 (4), 769-787.

Koumoutsakos, P., 2005. Multiscale flow simulations using particles. Annual Review of Fluid Mechanics 37, 457-487.

Lebedeva, N. A., Osiptsov, A. N., Sazhin, S. S., 2013. A combined fully Lagrangian approach to mesh-free modelling of transient two-phase flows. Atomization and Sprays 23 (1), 47-69.

Marble, F. E., 1970. Dynamics of dusty gases. Annual Review of Fluid Mechanics 2, 397-446.

Monaghan, J., 2012. Smooth particle hydrodynamics and its diverse applications. Annual Review of Fluid Mechanics 44, 323-346.

Ogami, Y., 1999. Simulation of heat-vortex interaction by the diffusion velocity method. ESAIM: Proc. 7, 313-324.

Osiptsov, A. N., 2000. Lagrangian modelling of dust admixture in gas flows. Astrophysics and Space Science 274 (1-2), 377-386. 
Osiptsov, A. N., Shapiro, Y. G., 1993. Heat transfer in the boundary layer of a gas-evaporating drops two-phase mixture. International Journal of Heat and Mass Transfer 36 (1), $71-78$.

Ould-Salihi, M. L., Cottet, G.-H., Hamraoui, M. E., May 2000. Blending finite-difference and vortex methods for incompressible flow computations. SIAM J. Sci. Comput. 22 (5), 1655-1674.

Ranz, W., Marshall, W., 1952. Evaporation from drops: Part 1. Chemical Engineering Progress 48 (3), $141-146$.

Sazhin, S. S., 2006. Advanced models of fuel droplet heating and evaporation. Progress in Energy and Combustion Science 32 (2), 162 - 214.

Sazhin, S. S., 2014. Droplets and Sprays. Springer.

Sazhin, S. S., Al Qubeissi, M., Nasiri, R., Gunko, V., Elwardany, A., Lemoine, F., Grisch, F., Heikal, M., 2014. A multi-dimensional quasidiscrete model for the analysis of diesel fuel droplet heating and evaporation. Fuel 129, $238-266$.

Sazhin, S. S., Shchepakina, E., Sobolev, V., 2010. Order reduction of a non-Lipschitzian model of monodisperse spray ignition. Mathematical and Computer Modelling 52 (34), 529 - 537.

Sazhina, E. M., Sazhin, S. S., Heikal, M. R., Babushok, V. I., Johns, R. J. R., 2000. A detailed modelling of the spray ignition process in diesel engines. Combustion Science and Technology 160 (1), 317-344.

Sbalzarini, I., Walther, J., Bergdorf, M., Hieber, S., Kotsalis, E., Koumoutsakos, P., 2006. PPM - a highly efficient parallel particlemesh library for the simulation of continuum systems. Journal of Computational Physics 215 (2), 566-588.

Walther, J., Koumoutsakos, P., 2001. Three-dimensional vortex methods for particle-laden flows with two-way coupling. Journal of Computational Physics 167 (1), $39-71$.

Wang, B. Y., Osiptsov, A. N., 2002. Mathematical modeling of near-wall flows of two-phase mixture with evaporating droplets. Applied Mathematics and Mechanics 23 (6), $647-654$. 
Yokota, R., Obi, S., 2010. Comparing vortex methods and finite difference methods in a homogeneous turbulent shear flow. International Journal for Numerical Methods in Fluids 63 (7), 828-846.
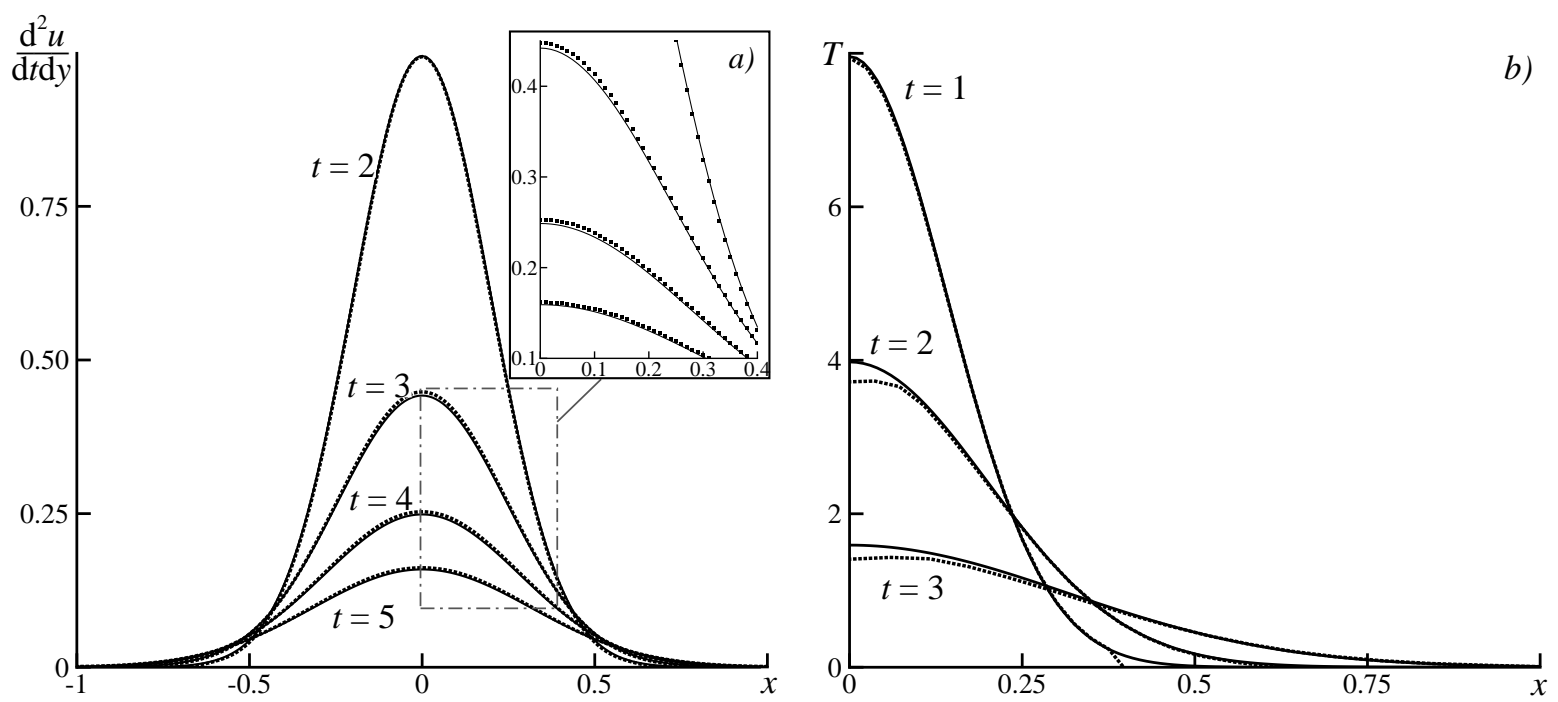

Figure 1: Comparison of the predictions of numerical (dotted) and analytical (solid) solutions for the Lamb vortex profiles at the $y=0$ cross-section: a) $\mathrm{d}^{2} u / \mathrm{d} t \mathrm{~d} y$; b) $T$. 


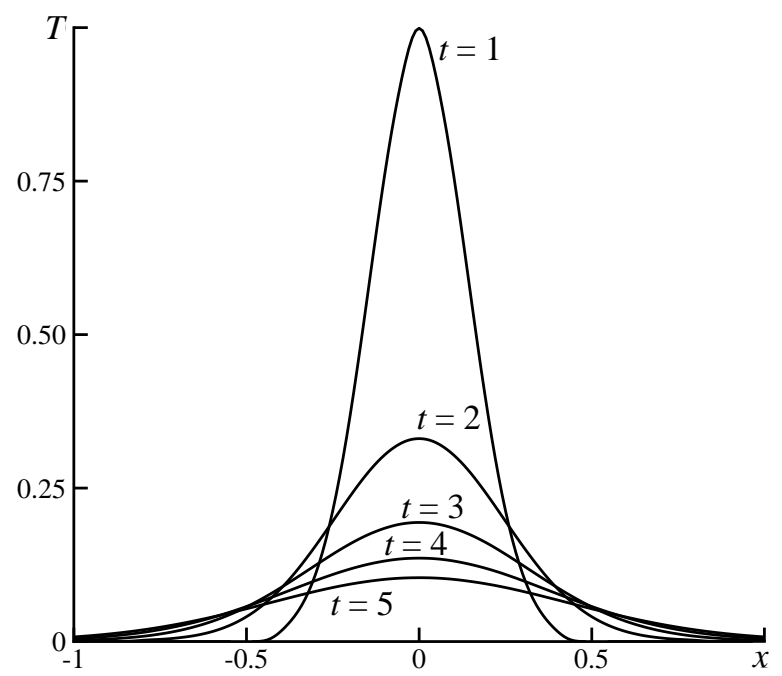

Figure 2: Evolution of non-dimensional gas temperature profiles for the Lamb vortex at the $y=0$ cross-section.

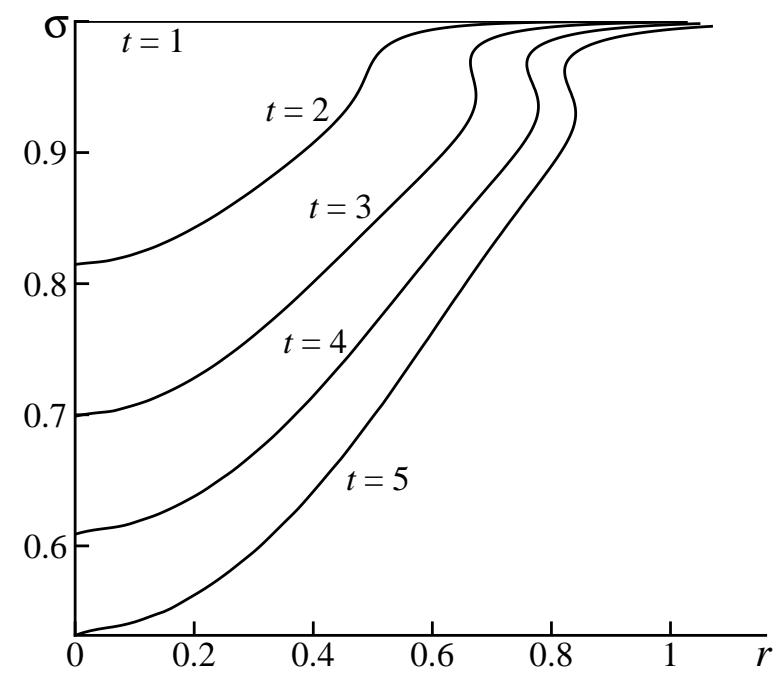

Figure 3: Time and space evolutions of non-dimensional droplet radii in the Lamb vortex. 


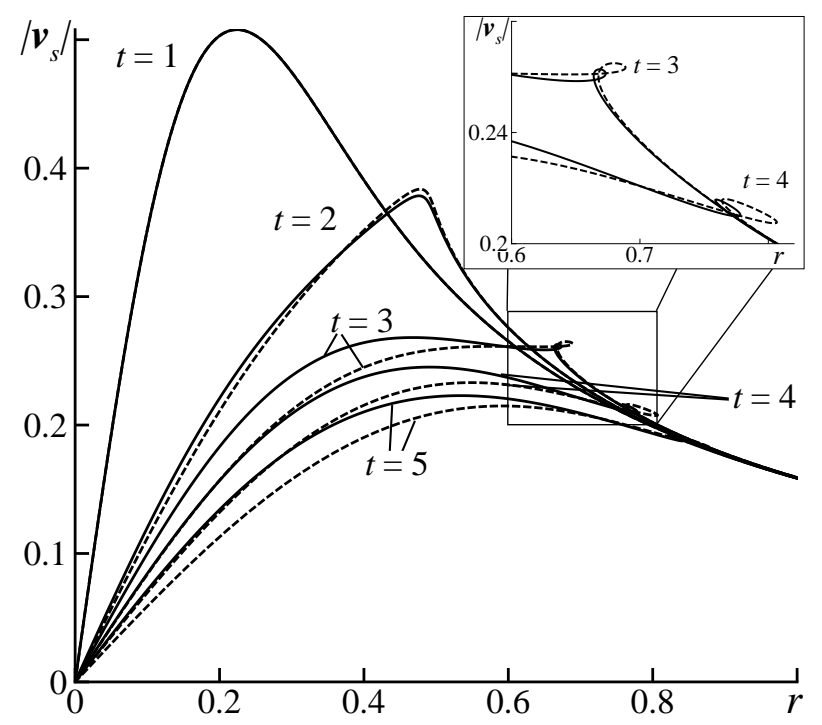

Figure 4: Time and space evolutions of the absolute value of velocity of a line of droplets in the Lamb vortex placed at $y=0$ at $t=t_{0}$ (solid curves - evaporating droplets, $\delta=-0.1$; dashed curves - non evaporating droplets).
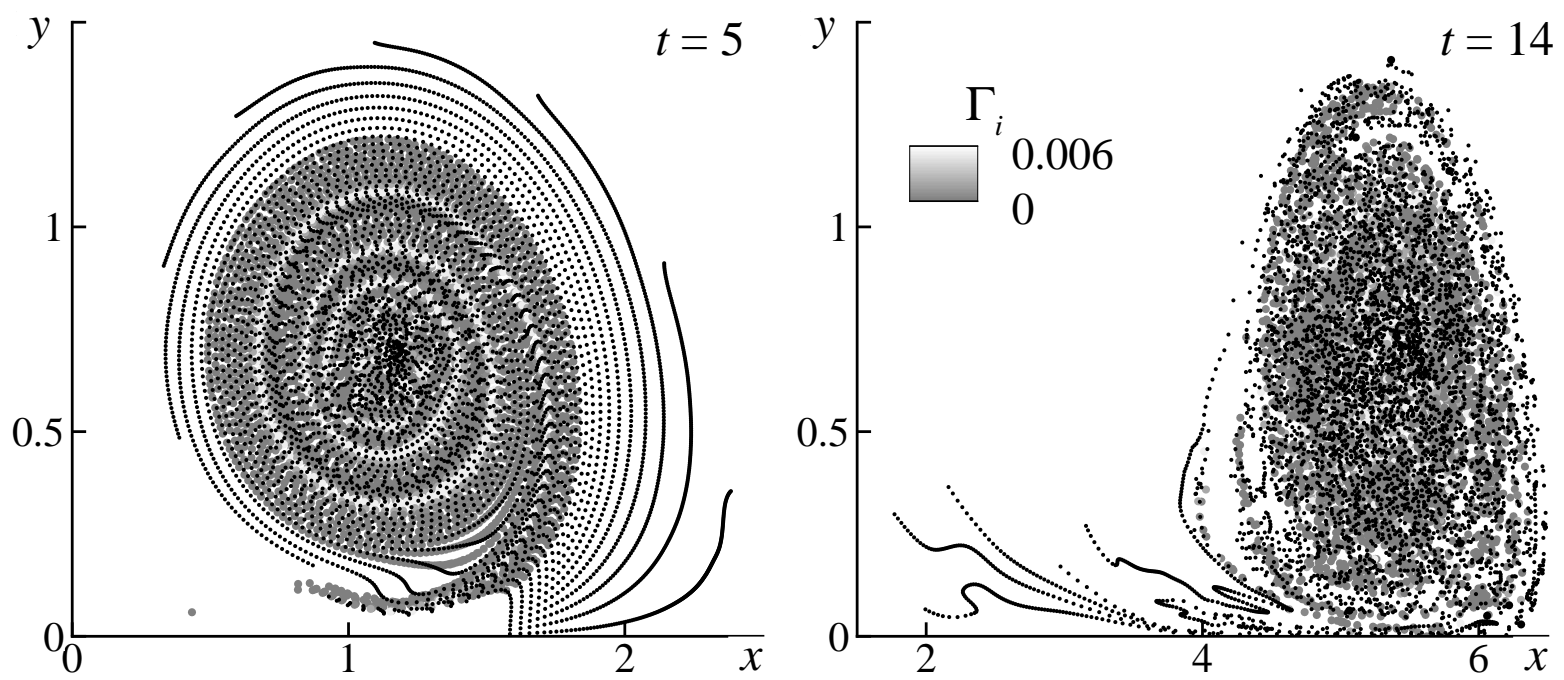

Figure 5: Blob distributions at time instants $t=5$ and $t=14$; black dots - thermal blobs; grey dots - viscous-vortex blobs, different levels of shading correspond to different strengths of blobs. Note that the viscous-vortex and thermal blobs move independently. 


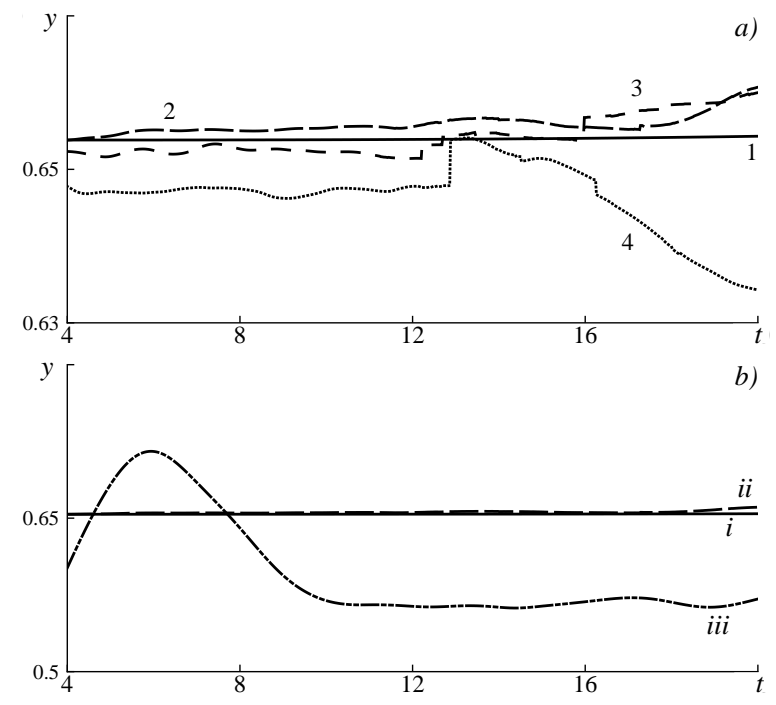

Figure 6: A vortex centre positions $\left(y_{V C}\right)$ calculated using 1 - analytical solution (Gaifullin and Zubtsov, 2004), 2 - 10000 viscous-vortex blobs, 3 - 6400 viscousvortex blobs, 4 - 3600 viscous-vortex blobs; b. (i) vortex centre position, asymptotic solution (Gaifullin and Zubtsov, 2004), (ii) vortex centre position, 10000 viscous-vortex blobs, (iii) thermal centre position, 10000 thermal blobs.

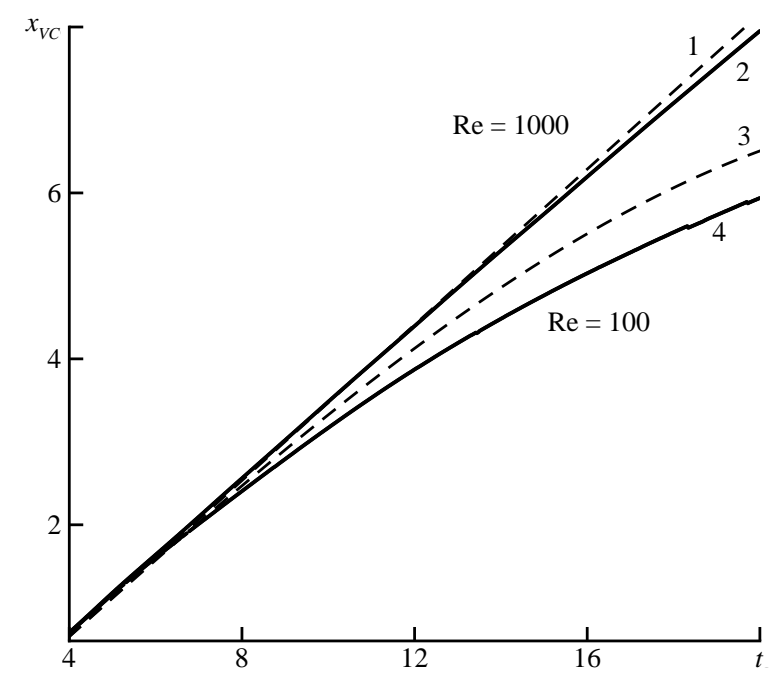

Figure 7: Vortex centre positions $\left(x_{V C}\right) ; 1,2-\operatorname{Re}=1000 ; 3,4-\operatorname{Re}=100 ; 1,3-$ predictions of the asymptotic solution (Gaifullin and Zubtsov, 2004); 2, 4 - predictions of the numerical simulations. 


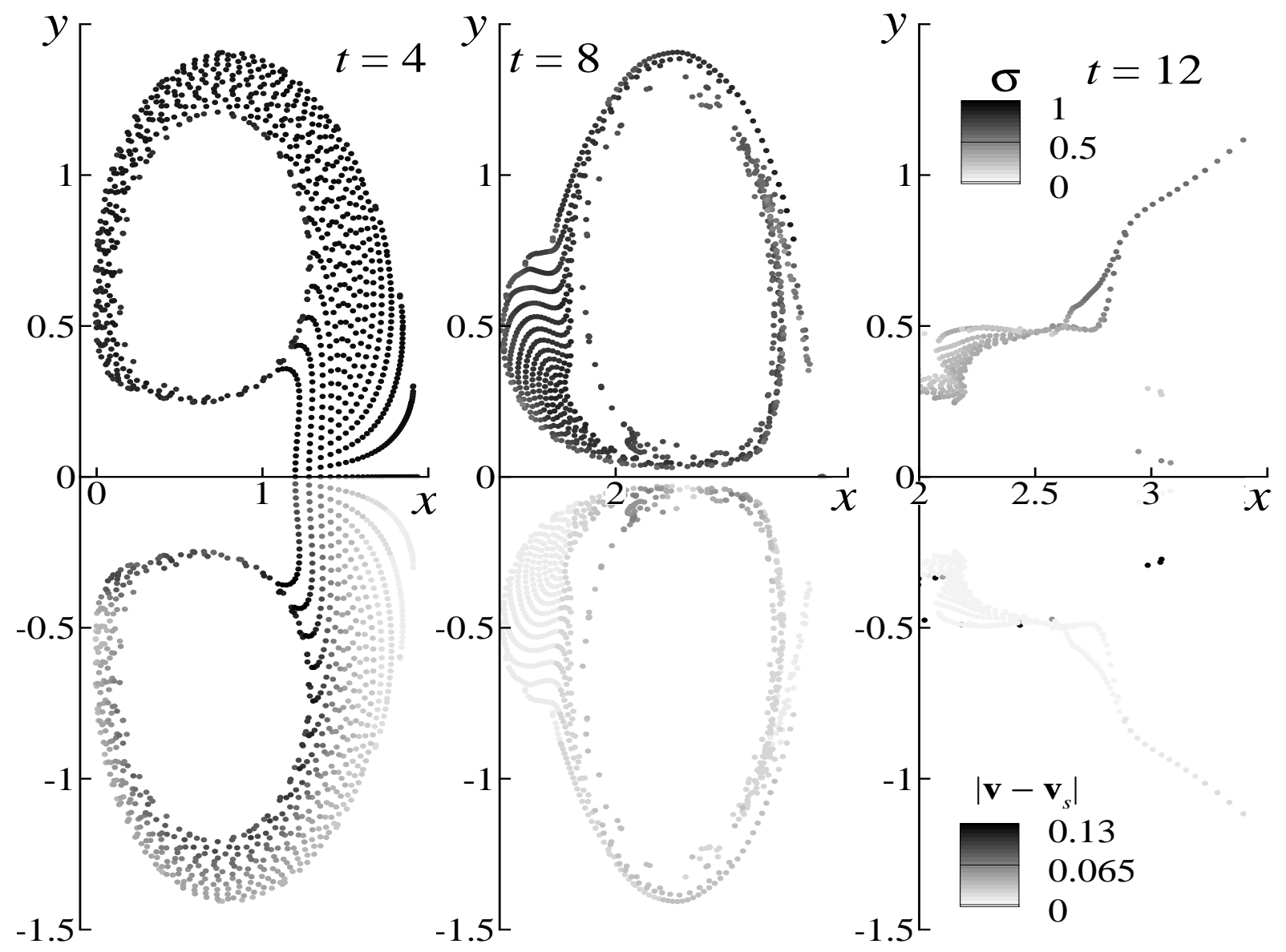

Figure 8: Droplet distributions, their sizes, and velocity slips at $t=4, t=8$ and $t=12$; $\beta=5.5, \delta=0.14$. 

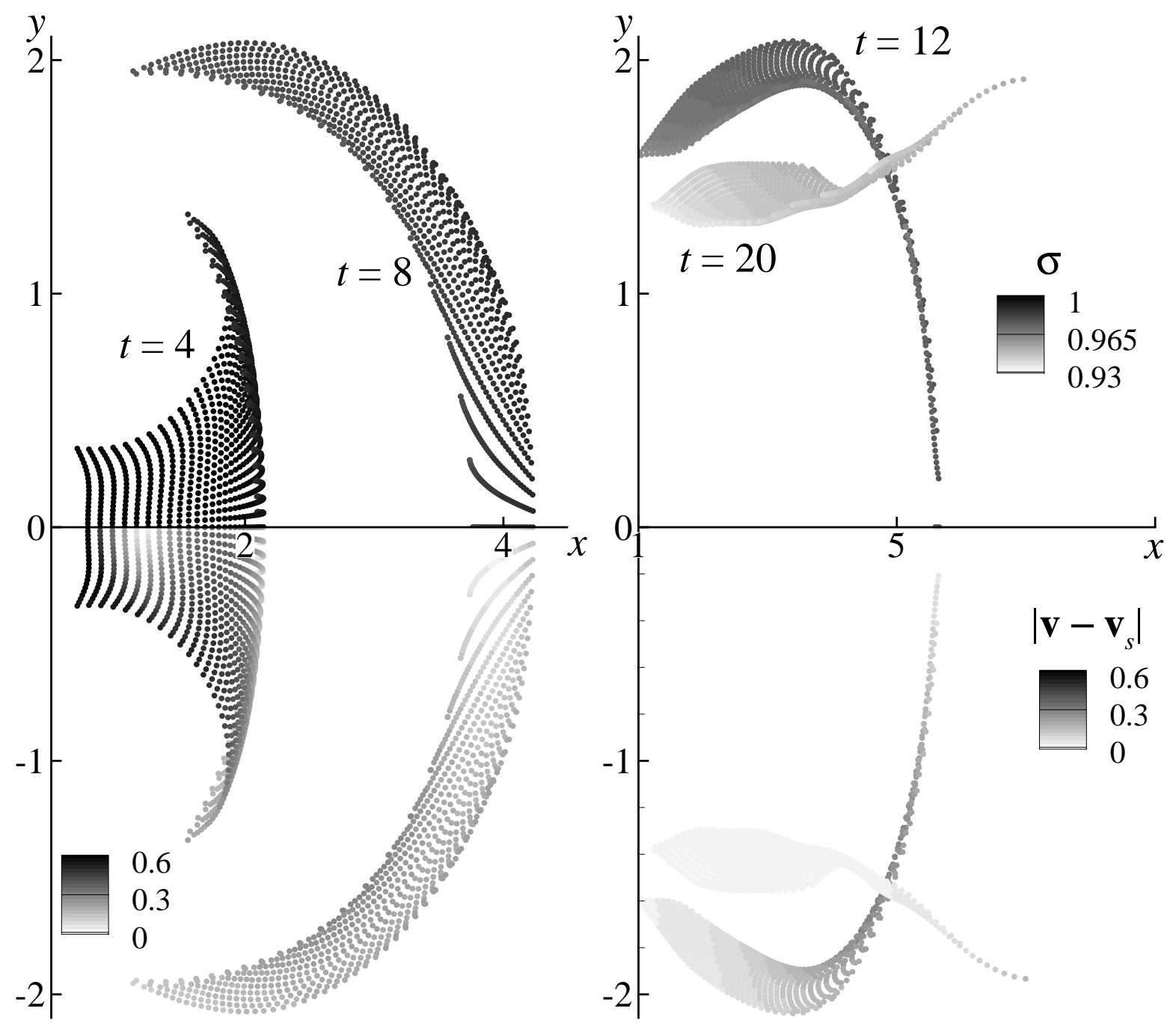

Figure 9: Droplet distributions, droplet diameters, and velocity slips at $t=4, t=8$, $t=12$, and $t=20 ; \beta=0.21, \delta=0.006$. 


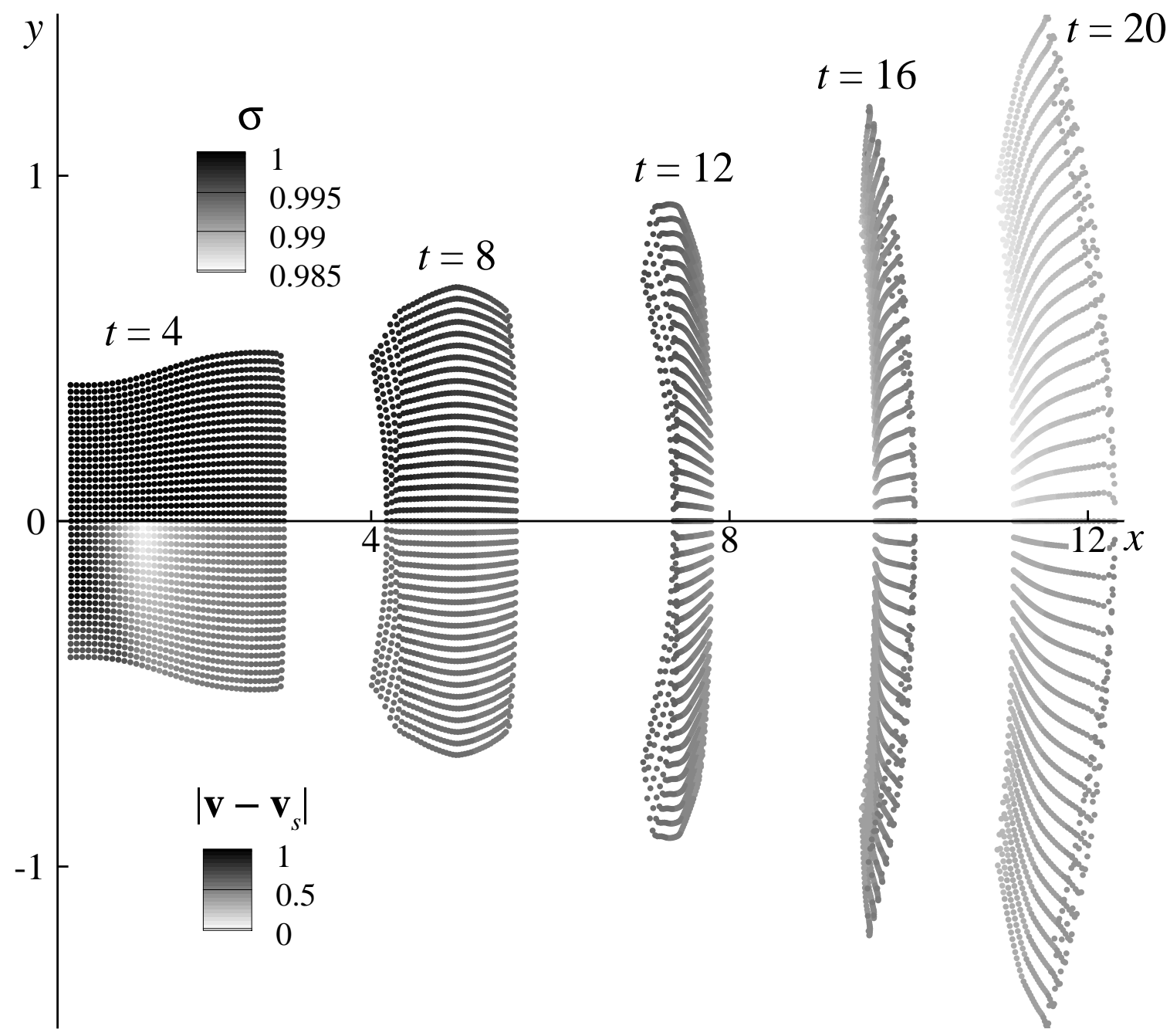

Figure 10: Droplet distributions, droplet diameters, and velocity slips at $t=4, t=8$, $t=12, t=16$, and $t=20 ; \beta=0.05, \delta=0.0014$. 